\title{
Sex differences in experimental measures of pain sensitivity and endogenous pain inhibition
}

This article was published in the following Dove Press journal:

Journal of Pain Research

29 June 2015

Number of times this article has been viewed

\author{
Hailey W Bulls' \\ Emily L Freeman' \\ Austen JB Anderson ${ }^{2}$ \\ Meredith T Robbins ${ }^{3}$ \\ Timothy J Ness ${ }^{3}$ \\ Burel R Goodin 1,3 \\ 'Department of Psychology, \\ University of Alabama at Birmingham, \\ Birmingham, AL, USA; ${ }^{2}$ Department \\ of Biology, Samford University, \\ Birmingham, AL, USA; ${ }^{3}$ Department of \\ Anesthesiology, University of Alabama \\ at Birmingham, Birmingham, AL, USA
}

Correspondence: Burel R Goodin Department of Psychology, University of Alabama at Birmingham, 1300 University Boulevard, Campbell Hall, Room 328,

Birmingham, AL 35294, USA

Tel +l 2059348743

Fax + I 205975 6II0

Email bgoodin I@uab.edu

\begin{abstract}
It has been suggested that increased pain sensitivity and disruption of endogenous pain inhibitory processes may account, at least in part, for the greater prevalence and severity of chronic pain in women compared to men. However, previous studies addressing this topic have produced mixed findings. This study examined sex differences in pain sensitivity and inhibition using quantitative sensory testing (QST), while also considering the influence of other important factors such as depressive symptoms and sleep quality. Healthy men $(n=24)$ and women $(n=24)$ each completed a QST battery. This battery included an ischemic pain task (IPT) that used a submaximal effort tourniquet procedure as well as a conditioned pain modulation (CPM) procedure for the assessment of endogenous pain inhibition. Prior to QST, participants completed the Center for Epidemiologic Studies Depression Scale and the Pittsburgh Sleep Quality Index. Analyses revealed significant sex differences for the ischemic pain task and the conditioned pain modulation procedure, such that women tolerated the ischemic pain for a shorter amount of time and demonstrated less pain inhibition compared with men. This remained true even when accounting for sex differences in depressive symptoms and sleep quality. The results of this study suggest that women may be more pain sensitive and possess less-efficient endogenous pain inhibitory capacity compared with men. Whether interventions that decrease pain sensitivity and enhance pain inhibition in women ultimately improve their clinical pain outcomes is an area of research that deserves additional attention in the future.
\end{abstract}

Keywords: sex differences, pain sensitivity, inhibition, depressive symptoms, sleep

\section{Introduction}

Chronically painful conditions are generally more prevalent in women than in men, from puberty to menopause and beyond. ${ }^{1,2}$ Common chronic pain conditions that are more prevalent in women than in men include migraine, fibromyalgia, irritable bowel syndrome, temporomandibular joint disorder, and pain associated with rheumatic diseases. ${ }^{3}$ Sex differences in the severity of the chronic pain conditions are also well documented. ${ }^{4,5}$ Women generally report more severe pain, more frequent bouts of pain, more anatomically diffuse pain, and longer-lasting pain than men with similar disease processes. ${ }^{6}$ Further, these sex differences persist even after male-specific and female-specific disorders (eg, male urologic and female gynecologic) are excluded from analyses.

Increased pain sensitivity and disruption of endogenous pain inhibitory processes have been hypothesized as potential mechanisms that may account, at least in part, for the greater prevalence and severity of chronic pain in women compared with men. ${ }^{7}$ Using quantitative sensory testing (QST) in laboratory settings, researchers 
have tested sex differences in response to experimental pain stimuli using unidimensional measures of pain sensitivity (eg, pain threshold and tolerance) as well as dynamic protocols of pain inhibition, such as conditioned pain modulation (CPM) tasks. ${ }^{8}$ The research addressing sex differences in unidimensional pain sensitivity measures has generally produced consistent results, such that women demonstrate lower thresholds and tolerances compared with men for pressure pain, electrical pain, ischemic pain, heat pain, and cold pain. ${ }^{9}$ Interestingly, studies investigating sex differences in dynamic tests of pain inhibitory processes such as CPM have produced inconsistent findings. ${ }^{10}$ Some studies have reported less-efficient pain inhibitory capacity for women compared to men, ${ }^{11-13}$ whereas other studies did not report any such sex differences. ${ }^{14-17}$ Given the mixed nature of the literature regarding sex differences in pain inhibition, additional research addressing this topic seems warranted.

When examining sex differences in pain sensitivity and endogenous inhibition, it is important to simultaneously consider other psychological and behavioral factors known to influence the experience of pain. Doing so can help ensure more accurate effect-size estimation of any resultant sex differences. Depressive symptoms and sleep quality are two clinically relevant examples of psychological and behavioral factors, respectively, that have well-documented relationships with clinical pain. ${ }^{18,19}$ Furthermore, experimental evidence suggests that depressive symptoms and sleep quality can alter pain sensitivity as well as pain inhibitory capacity. ${ }^{20-26}$ A noteworthy shortcoming of the previous research that specifically investigated sex differences in endogenous pain inhibition has been the lack of consideration for influential psychological and behavioral factors like depressive symptoms and sleep quality, which may have biased results.

Considering this shortcoming, the current study recruited a sample of healthy, chronic-pain-free adults equally comprising men and women. Each study participant provided self-report regarding recent depressive symptoms and sleep quality prior to completing a QST battery. The QST battery included induced ischemia for the assessment of unidimensional pain sensitivity and CPM as a dynamic model of endogenous pain inhibition. The goal was to examine sex differences across the various QST measures, while also accounting for the relative influences of depressive symptoms and sleep quality. It was specifically hypothesized that: 1) women would demonstrate greater ischemic pain sensitivity and less CPM compared with men, and 2) these differences between men and women would remain significant even after accounting for depressive symptoms and sleep quality.

\section{Methods Overview}

Data from this study were combined with the baseline data from a separate study that evaluated the influence of intranasal oxytocin on experimental pain sensitivity, endogenous pain inhibition, and mood states. ${ }^{27}$ Inclusion criteria for the two studies were identical, as were the pain testing procedures. Because only the baseline data collected as part of this oxytocin study were used, there was therefore no effect from the oxytocin administration. Combining these data is further justified in that identical protocols for evaluating pain sensitivity and endogenous pain inhibition were used across studies. This study was conducted in accordance with the Declaration of Helsinki ethical principles for medical research. Each protocol was approved by the University of Alabama at Birmingham Institutional Review Board, in accordance with ethical research conduct guidelines. Written informed consent was obtained from participants and they were compensated for their involvement.

\section{Participants}

The final combined sample consisted of 48 young, healthy adults recruited from the University of Alabama at Birmingham campus via flyers. The data included in this study were collected between February 2013 and June 2014. Potential participants were screened via telephone to determine study eligibility. Inclusion criteria for participation included 1) age between 19-45 years, 2) no ongoing chronic pain problems, 3 ) no episodes of acute pain within 2 weeks prior to study participation, 4) no diagnosis of hypertension or use of medications for blood pressure, 5) no circulatory disorders, 6) no history of cardiac events, 7) no history of metabolic disease or neuropathy, 8) no current use of prescription medications, including analgesics, tranquilizers, antidepressants, or other centrally acting agents, 9) no diagnosed mental health disorders, 10) no current pregnancy, 11) no liver or kidney disease, and 12) no disorders involving the neuroendocrine system. Sessions were to be rescheduled if any participant reported use of alcohol, opioid pain medications, or nonsteroidal antiinflammatory drugs in the 24 hours prior to their appointment; however, this was not necessary for any participant.

\section{Procedures}

At the beginning of each study session, height and weight were measured to assess body mass index. Resting blood 
pressure was taken three times, which ensured that none of the participants were hypertensive. Questionnaires assessing depressive symptoms and sleep quality were also completed prior to the initiation of pain testing. Participants then completed a QST battery that included an ischemic pain task (IPT) and a CPM task with pressure as the test stimulus and cold water as the conditioning stimulus.

\section{Quantitative Sensory Testing}

Ischemic pain task

Participants completed the IPT first to assess pain sensitivity. The IPT consisted of a modified submaximal effort tourniquet procedure. ${ }^{28}$ The hand was exercised while blood flow to the arm was occluded, resulting in ischemic pain. Per standard procedure, $50 \%$ of maximum grip strength of the dominant hand was determined using a Lafayette handheld dynamometer (Lafayette Instrument Company, Lafayette, IN, USA). Before occlusion, participants elevated the dominant arm above heart level for 30 seconds, draining blood from the arm. A blood pressure cuff positioned proximal to the elbow was then inflated to $240 \mathrm{mmHg}$, using a Hokanson E20 rapid cuff inflator (DE Hokanson, Inc., Bellevue, WA, USA). Inflation of the cuff occluded blood flow to the arm, and the arm was lowered to resting position. Participants performed 20 handgrip exercises consisting of 2 seconds grip, 4 seconds rest at $50 \%$ of their maximum grip strength. Ratings of pain intensity and unpleasantness were provided at 30-second intervals by instructing participants to rate their pain on $0-100$ numeric rating scales, where $0=$ "No pain" and $100=$ "Pain as intense (or unpleasant) as I can imagine." ${ }^{29}$ Instructions were given to say the word "stop" if the pain had become intolerable. The IPT was completed either when the participant discontinued due to the pain or at 900 seconds. Pain intensity and unpleasantness ratings were calculated by averaging the respective ratings across the entirety of the IPT. The duration of the exposure was recorded and classified as pain tolerance, regardless of whether the participant completed the entire task or terminated the IPT prior to the allotted maximum time of exposure.

\section{Cold pressor tasks}

Participants completed consecutive cold pressor tasks by immersing their nondominant hand up to the wrist, with an open hand, in the cold water for up to 1 minute per immersion. ${ }^{30}$ Five-minute rest periods were observed between each immersion. The cold pressor tasks were always presented in descending order using the following temperatures: $16^{\circ} \mathrm{C}$, $12^{\circ} \mathrm{C}, 8^{\circ} \mathrm{C}$, and $5^{\circ} \mathrm{C}$. The water temperatures were maintained within $\pm 0.05^{\circ} \mathrm{C}$ by a refrigeration unit (Neslab, Portsmouth, $\mathrm{NH}$, USA), which circulated the water continuously to avoid localized warming around the hand. Pain intensity was recorded at 30 seconds and 60 seconds, using the $0-100$ scale. If the participant removed their hand from the water before 60 seconds was completed, they provided a rating of pain intensity at the time of removal. These cold pressor tasks were performed to determine individually tailored temperatures for later use as the conditioning stimulus in the CPM task. Thus, the cold water temperature that elicited a pain intensity rating of moderate pain ( $\sim 50$, range $40-60)$ on the $0-100$ scale was selected for CPM use.

\section{Conditioned pain modulation task}

CPM was assessed after an additional 5-minute rest period using a heterotopic noxious conditioning stimulation paradigm. ${ }^{31}$ In this task, algometric assessment on the dominant dorsal forearm provided the phasic test stimulus, while the cold pressor task was used for the tonic conditioning stimulus. A handheld algometer (Somedic AB, Horby, Sweden) was applied to the dorsal forearm three times to determine participant's baseline pressure pain threshold (PPTs). Participants were instructed to indicate when the increasing pressure sensation first became painful. PPTs were measured in kilopascals. After the PPT baseline tests, participants underwent a series of tailored cold pressor tasks using the cold water temperature that evoked a moderate level of pain. Approximately 30 seconds into the cold water immersion, while the hand remained in the water, the algometer was again used to deliver noxious mechanical stimulation to the opposite dorsal forearm. This procedure was repeated twice with a 2-minute rest period between trials. Like before, participants indicated when the increasing pressure stimulation first became painful. This represented their conditioned PPTs. For the analysis of CPM, we followed recommendations for presenting results and calculation of CPM using the percent change..$^{32}$ Percent change for PPTs within each trial was calculated (and averaged) according to the following formula:

$$
\text { (Conditioned PPTs }- \text { test PPTs) / test PPTs }) \times 100
$$

\section{Questionnaires}

\section{Center for Epidemiologic Studies Depression Scale}

The Center for Epidemiologic Studies Depression Scale (CES-D) is a 20-item self-report tool that measures symptoms of depression in the past week. ${ }^{33}$ Questions evaluate depressed mood, guilt/worthlessness, helplessness/ 
hopelessness, psychomotor retardation, loss of appetite, and sleep disturbance. The CES-D has previously been used in a variety of research groups, including both psychiatric and nonpsychiatric samples as well as clinical samples with medical illness. ${ }^{34}$ The total score ranges from $0-60$, and this single total score was used in this study as an estimate of the degree of individuals' depressive symptomatology. When the CES-D is used specifically in young, healthy adults, its validity and internal consistency have been reported to be acceptable. ${ }^{33}$

\section{Pittsburgh Sleep Quality Index}

The Pittsburgh Sleep Quality Index (PSQI) is a self-rated questionnaire that retrospectively assesses sleep quality and disturbances over the past month. ${ }^{35}$ Nineteen individual items generate seven "component" scores: subjective sleep quality, sleep latency, sleep duration, habitual sleep efficiency, sleep disturbances, use of sleep medications, and daytime dysfunction. Each of the seven component scores is weighted equally on a scale from $0-3$, with 0 indicating no difficulty and 3 indicating severe difficulty. The sum of scores for these seven component scores yields one global score, ranging from 0-21. Higher scores indicate worse sleep quality, and a global PSQI score $>6$ is consistent with poor sleep quality. ${ }^{36}$ The seven component scores of the PSQI have been shown to possess good internal consistency, and the overall global score has demonstrated good test-retest reliability. ${ }^{35,36}$

\section{Data analysis}

All data were analyzed using SPSS, version 22 (IBM Corporation, Armonk, NY, USA). All participants provided complete demographic, questionnaire, and QST data. Descriptive statistics were computed for the overall sample as well as separately for men and women; data are presented as percentages or as means and standard deviations (SD). Relationships among continuously measured variables were tested using Pearson correlation coefficients, while relationships among categorical variables were tested with Chi-square. Analysis of variance was used to determine the presence of significant sex differences in depressive symptoms and sleep quality. Lastly, a series of two separate multivariate analysis of covariance (MANCOVA) were completed to evaluate sex differences in pain sensitivity and endogenous pain inhibition while controlling for depressive symptoms as well as sleep quality. Specifically, IPT tolerance and ratings of pain intensity and pain unpleasantness were included in the first MANCOVA. In the second MANCOVA, baseline PPTs, conditioned PPTs, and percent change in CPM were included.
To evaluate significant sex differences revealed by either MANCOVA, the univariate $F$ statistics were examined to determine the specific aspects of pain sensitivity and endogenous pain inhibition upon which men and women significantly differed.

\section{Results}

\section{Characteristics of the study sample}

Characteristics of the 48 healthy young adults who were included in this study are presented in Table 1 . The mean age was 22.81 years (SD 4.60), with an equal number of men and women participating. The primary ethnicity represented was Caucasian (54\%). Resting blood pressures fell within the normotensive to prehypertensive ranges on average; the mean body mass index was $26.33 \mathrm{~kg} / \mathrm{m}^{2}$ (SD 5.70). Neither acute pain in the 2 weeks preceding the study session nor a history of chronic pain was reported by any of the participants. None reported taking any prescribed or overthe-counter pain medications prior to study involvement. All participants were either college graduates or were in the process of obtaining their college degrees. Of the women who participated, six of $24(25 \%)$ reported use of oral birth control medication.

\section{Covariates}

It is important to reiterate that data from this study were combined with the baseline data of a separate study previously conducted by our group. ${ }^{27}$ Notably, none of the IPT pain sensitivity variables (eg, tolerance, pain intensity, pain unpleasantness) or CPM results differed significantly as a function of the study from which the data were originally collected (all $P>0.05$ ). This suggests that, when combined,

Table I Participant characteristics

\begin{tabular}{|c|c|c|c|}
\hline Characteristic & Overall, $n=48$ & Men, $n=24$ & Women, $n=24$ \\
\hline Age (years), mean (SD) & $22.81(4.60)$ & $24.54(5.03)$ & $2 I .08(3.4 I)$ \\
\hline BMI, mean (SD) & $26.33(5.70)$ & $26.33(4.88)$ & $26.33(6.53)$ \\
\hline \multicolumn{4}{|c|}{ Resting BP (mmHg), mean (SD) } \\
\hline Systolic & $123.63(12.74)$ & I30.44 (10.35) & II6.8I (1I.29) \\
\hline Diastolic & $75.10(9.79)$ & 76.40 (11.57) & 73.81 (11.29) \\
\hline \multicolumn{4}{|c|}{ Ethnic background, n (\%) } \\
\hline Caucasian & $26(54.2)$ & $17(70.8)$ & $9(37.5)$ \\
\hline African American & $16(33.3)$ & $5(20.8)$ & II (45.8) \\
\hline $\begin{array}{l}\text { Asian/Pacific } \\
\text { Islander }\end{array}$ & $4(8.3)$ & I (4.2) & $3(12.5)$ \\
\hline Hispanic & $2(4.2)$ & $\mathrm{I}(4.2)$ & I (4.2) \\
\hline \multicolumn{4}{|l|}{ Education, n (\%) } \\
\hline College graduate & $8(16.7)$ & $20(83.3)$ & $0(0)$ \\
\hline In college & $40(83.3)$ & $4(16.7)$ & $24(100)$ \\
\hline
\end{tabular}

Abbreviations: $\mathrm{BMI}$, body mass index; BP, blood pressure; $\mathrm{SD}$, standard deviation. 
the results formed a homogeneous set of data from which sex differences in pain sensitivity and endogenous pain inhibition could be examined. Due to this homogeneity, it was not necessary to statistically adjust our analyses according to the original study from which the data were generated.

Results revealed significant sex differences in depressive symptoms and sleep quality (Table 2 ). Women reported greater severity of depressive symptoms $(F=5.90, P=0.019)$ as well as poorer sleep quality $(F=7.06, P=0.011)$ compared with men. Depressive symptoms and sleep quality were significantly correlated; however, neither was significantly correlated with any of the IPT pain sensitivity variables or CPM (Table 3). Despite the lack of significant correlations, both depressive symptoms and sleep quality were included as covariates in subsequent analyses examining sex differences in IPT pain sensitivity and CPM.

\section{IPT pain sensitivity}

Mean values for the IPT pain sensitivity variables are displayed in Table 4 for the overall study sample as well as separately for men and women. The first MANCOVA revealed significant sex differences for IPT pain sensitivity while controlling for depressive symptoms and sleep quality $(\lambda=0.793, F=3.67, P=0.020)$. The follow up univariate $\mathrm{F}$ statistics showed there was a significant sex difference for IPT tolerance time $(F=10.48, P<0.01)$ but not for ratings of pain intensity or pain unpleasantness. Although women tended to discontinue the IPT after a significantly shorter amount of time than did men, their ratings of pain intensity and pain unpleasantness were comparable (Figure 1A and $\mathrm{B})$.

\section{CPM effect at the forearm}

Of the four cold water intensities used for tailoring the conditioning pain stimulus, the majority of participants used $12^{\circ} \mathrm{C}$ as their conditioning stimulus to achieve a moderate amount of pain $(\mathrm{n}=28,58 \%)$. Otherwise, $16^{\circ} \mathrm{C}$ was utilized for five individuals $(10 \%), 8^{\circ} \mathrm{C}$ was utilized for eight individuals $(17 \%)$, and $5^{\circ} \mathrm{C}$ for the remaining seven (15\%). CPM did not significantly differ as a function of the temperature used to tailor the cold water conditioning

Table 2 Depressive symptoms and sleep quality

\begin{tabular}{llll}
\hline Variables & $\begin{array}{l}\text { Overall, } \\
\text { mean (SD) }\end{array}$ & $\begin{array}{l}\text { Men, } \\
\text { mean (SD) }\end{array}$ & $\begin{array}{l}\text { Women, } \\
\text { mean (SD) }\end{array}$ \\
\hline CES-D & $10.10(6.68)$ & $7.88(5.91)$ & $12.33(6.77)$ \\
PSQI & $4.90(2.14)$ & $4.13(1.42)$ & $5.67(2.46)$ \\
\hline
\end{tabular}

Abbreviations: CES-D, Center for Epidemiologic Studies Depression Scale; PSQI, Pittsburgh Sleep Quality Index; SD, standard deviation.
Table 3 Pearson correlations

\begin{tabular}{lllllll}
\hline Variable & $\mathbf{I}$ & $\mathbf{2}$ & $\mathbf{3}$ & $\mathbf{4}$ & $\mathbf{5}$ & $\mathbf{6}$ \\
\hline I. Sex & & & & & & \\
2. CES-D & $0.34^{*}$ & - & & & & \\
3. PSQI & $0.37^{*}$ & $0.47^{* *}$ & - & & & \\
$\begin{array}{l}\text { 4. IPT tolerance } \\
\text { 5. IPT pain }\end{array}$ & $-0.43^{* *}$ & -0.11 & -0.05 & - & & \\
$\quad$ intensity & 0.21 & 0.16 & 0.13 & -0.12 & - & \\
6. IPT pain & 0.17 & 0.14 & 0.09 & -0.18 & $0.8 I^{* *}$ & - \\
$\quad$ unpleasantness & & & & & & \\
7. CPM & $-0.30^{*}$ & -0.19 & -0.27 & -0.04 & $-0.30^{*}$ & -0.24 \\
\hline
\end{tabular}

Note: $* P<0.05 ; * * P<0.01$; sex coded as $\mathrm{I}=$ men, $2=$ women.

Abbreviations: CES-D, Center for Epidemiologic Studies Depression Scale; CPM, conditioned pain modulation; IPT, ischemic pain task; PSQI, Pittsburgh Sleep Quality Index.

stimulus $(F=1.99, P=0.129)$. Furthermore, men and women did not significantly differ in the temperatures of the cold water needed to achieve a moderate amount of pain $\left(\chi^{2}=6.13\right.$, $P=0.106)$. This suggests that any sex difference in CPM is likely to be independent of the intensity of the tailored cold water conditioning stimulus.

Mean values for CPM are displayed in Table 4 for the overall study sample as well as separately for men and women. The second MANCOVA revealed significant sex differences for CPM while again controlling for depressive symptoms and sleep quality $(\lambda=0.696, F=6.11, P=0.002)$. Follow-up univariate $F$ statistics showed significant sex differences for baseline PPTs $(F=6.45, P=0.006)$, conditioned PPTs $(F=14.43, P<0.001)$, and CPM ( $F=5.53, P=0.023)$. Women demonstrated significantly lower baseline and conditioned PPTs than men as well as significantly less CPM at the forearm according to percent change. These results

Table 4 Pain sensitivity responses to the IPT and baseline and conditioned PPTs during concurrent cold water immersion to determine the magnitude of CPM at the forearm

\begin{tabular}{llll}
\hline Variables & $\begin{array}{l}\text { Overall, } \\
\text { mean (SD) }\end{array}$ & $\begin{array}{l}\text { Men, } \\
\text { mean (SD) }\end{array}$ & $\begin{array}{l}\text { Women, } \\
\text { mean (SD) }\end{array}$ \\
\hline IPT & & & \\
$\quad \begin{array}{l}\text { Tolerance } \\
\text { (seconds) }\end{array}$ & $446.65(225.68)$ & $543.54(242.38)$ & $349.75(160.43)$ \\
$\quad \begin{array}{l}\text { Pain intensity } \\
\text { Pain }\end{array}$ & $48.97(20.88)$ & $44.60(19.57)$ & $53.34(21.63)$ \\
$\quad 54.75(20.35)$ & $51.29(18.65)$ & $58.21(21.75)$ \\
unpleasantness & & & \\
$\begin{array}{l}\text { CPM } \\
\text { Conseline PPTs }\end{array}$ & $357.25(155.36)$ & $411.15(159.93)$ & $303.35(132.98)$ \\
$\begin{array}{l}\text { PPTs } \\
\text { CPM }\end{array}$ & $416.79(188.49)$ & $506.71(193.78)$ & $326.86(134.81)$ \\
(\% change) & $18.72(29.03)$ & $27.46(33.78)$ & $9.98(20.55)$ \\
\hline
\end{tabular}

Note: All PPT values are presented as kilopascals $(\mathrm{kPa})$.

Abbreviations: CPM, conditioned pain modulation; IPT, ischemic pain task; PPT, pressure pain threshold; SD, standard deviation. 

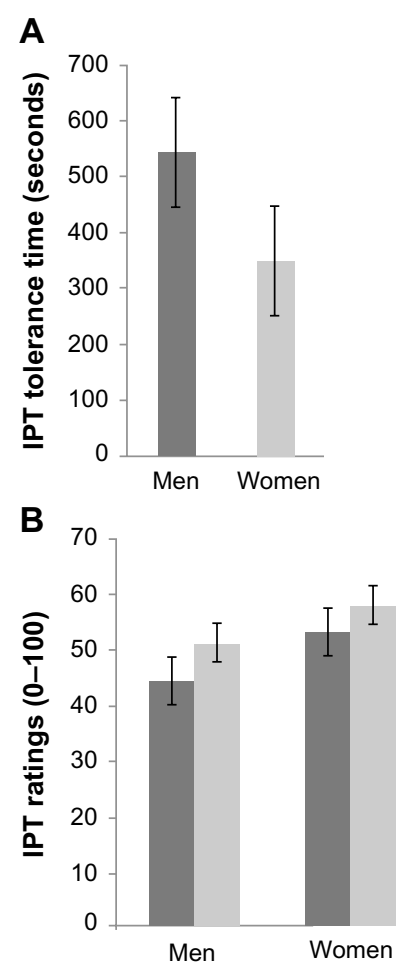

Figure I Differences in pain sensitivity between men and women in response to the IPT.

Notes: (A) IPT tolerance time; (B) ratings of pain intensity and pain unpleasantness. Abbreviation: IPT, ischemic pain task.

suggest that women may have less-efficient endogenous pain inhibition than men (Figure 2A and B).

\section{Discussion}

The results of the present investigation provide evidence for sex differences in pain sensitivity as well as endogenous pain inhibition. Specifically, women demonstrated less tolerance for the IPT and less efficient CPM compared to men. A unique finding from this study is that sex differences in both IPT tolerance and CPM effects continued even after considering the influences of depressive symptoms and sleep quality. The sex difference in IPT tolerance revealed in this study generally parallels results from a prior investigation that examined unidimensional aspects of pain sensitivity such as threshold and tolerance. ${ }^{37}$ Our finding of a sex difference in CPM effects is also consistent with several past studies that have shown less-efficient pain inhibitory capacity for women compared with men. ${ }^{1-13}$ Interestingly, of the limited number of studies published to date, the majority seemingly argue against sex differences in endogenous pain inhibitory processes. ${ }^{10}$ The mixed nature of these findings may be attributed in part to differences in experimental methodology as well as the intensity of the conditioning stimulus used to assess inhibitory effects. ${ }^{38}$ For example, previous studies
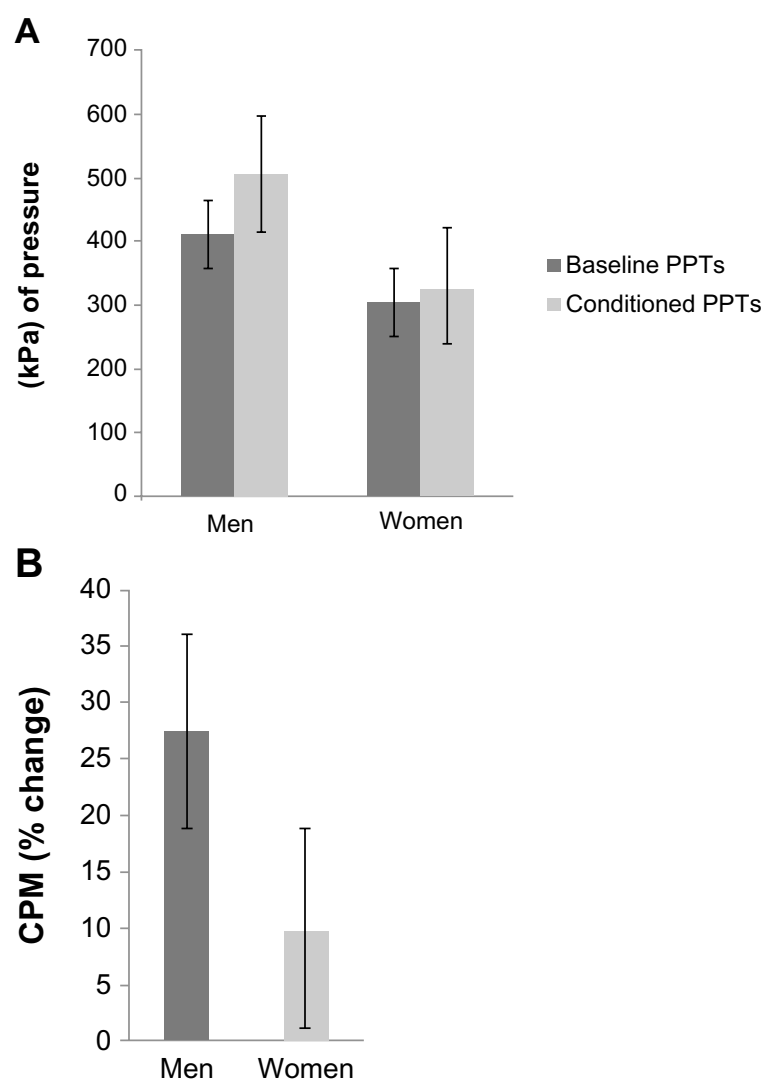

Figure 2 Differences in endogenous pain inhibition between men and women. Notes: (A) Baseline PPTs and conditioned PPTs; (B) CPM at the forearm. Abbreviations: CPM, conditioned pain modulation; PPT, pressure pain threshold.

examining sex differences in CPM tended to more consistently reveal greater CPM for men than for women when CPM was assessed via pain thresholds. ${ }^{10}$ This is consistent with our findings and the methodology used in this study for the assessment of CPM.

Much attention has been directed towards explaining the biological underpinnings of sex differences in pain sensitivity. For example, it has been suggested that sex chromosomes and steroidal actions on development lead to differences in pain physiology between men and women. ${ }^{39,40}$ Work in both animal ${ }^{41,42}$ and human ${ }^{43,44}$ models also suggests that sex-specific hormones (eg, estrogen) influence sex-specific responses to pain. However, the exact mechanism of these effects in development and/or adulthood is debated. ${ }^{39,40,45,46}$ Further investigations have identified neurobiological differences between men and women in brain regions related to pain sensitivity, such as the periaqueductal gray ${ }^{47}$ and the strength of its connectivity with the amygdala caudate and the putamen. ${ }^{48}$ Despite efforts to characterize this phenomenon from a biological perspective, nothing to date has fully accounted for sex differences in pain sensitivity. Thus, further research into the underlying physiology of 
sex-specific pain sensitivity is necessary, particularly in human models.

Significant sex differences were also observed in depressive symptoms and sleep quality. Consistent with our hypotheses, the addition of depressive symptoms and sleep quality as covariates did not result in any significant diminution of the sex differences found for IPT tolerance and CPM. As such, our results suggest that participants' sex was the more important factor contributing to the experience of pain in both unidimensional (IPT) and dynamic (CPM) tests. This is not to say that depressive symptoms and sleep quality do not meaningfully influence pain sensitivity and endogenous pain inhibition. It is important to interpret these findings in light of the fact that our study sample comprised young and healthy participants. Thus, pervasiveness of depressive symptoms and poor sleep quality was likely diminished in this study sample, which did not exhibit any appreciable major depression or insomnia. In contrast to our findings, negative emotional states have been oft-researched in the context of experimental pain responses, with previous results suggesting that depressive symptoms can alter both pain sensitivity and endogenous pain inhibition. ${ }^{49}$ Sleep has also recently been shown to influence experimental pain responses, with poor sleep quality resulting in lessened pain inhibition. ${ }^{50}$ In groups of clinical populations with greater prevalence of major depression and insomnia, these factors may play more of a central role in the differential pain experiences of men and women. ${ }^{18,19}$

Understanding how different groups of people experience pain is crucial in order to enhance pain assessment and treatment by allowing providers to personalize pain medicine based on the sources of this variation. Specifically studying how pain is experienced among men and women seems worthwhile because they represent major population groups that show significant differences in pain-related phenotypes. ${ }^{51}$ Also, the influence of sex on pain responses is driven by complex interactions among multiple pain-relevant biological, psychological, behavioral, and sociocultural factors. ${ }^{52}$ This study included two important psychological and behavioral factors: depressive symptoms and sleep quality. Although neither demonstrated appreciable relationships with IPT tolerance or CPM in this healthy sample, both factors were found to differ significantly between men and women. Women reported greater depressive symptoms and poorer sleep quality than men. Given the known deleterious health consequences of depression and poor sleep among people experiencing chronic pain, ${ }^{53,54}$ additional consideration of whether depression and poor sleep might help to explain differences in clinical pain outcomes between men and women seems warranted.

Because relatively few human studies have addressed sex differences in endogenous pain inhibitory processes,${ }^{10}$ it is not yet possible to conclude that clinically relevant differences in CPM exist between men and women. However, the potential clinical implications of our findings, particularly for women, are underscored by previous research that has related responses to QST to clinical pain outcomes. ${ }^{55}$ For instance, it has been shown that greater pain tolerance and more efficient endogenous pain inhibition were associated with less clinical pain and better physical functioning. ${ }^{56,57}$ Further, deficient pain-inhibitory capacity has been shown to be predictive of postoperative chronic pain development and severity. ${ }^{58}$ It has been suggested that responses to experimental pain testing may be better related to the clinical pain experiences of women compared with men. ${ }^{7}$ As previously described, women often experience a greater prevalence and severity of chronic pain across a variety of clinical conditions relative to men, and it may be that less-efficient endogenous pain inhibition plays a contributory role. Additional research on this topic is needed to confirm or refute such a hypothesis.

Several limitations must be considered when interpreting these findings. First, as noted previously, this study included a healthy sample of young adults without any known psychiatric or sleep-related disorders. This may have resulted in subclinical severity of responses for depressive symptoms and problems with sleep quality. Indeed, this limits our ability to generalize these findings to clinical pain populations who would likely have greater severity of depressive symptoms and poorer sleep quality. Second, it was not feasible for the sex of the experimenters to be matched to the sex of the participants. A scant amount of previous literature has shown that pain responses to QST may differ according to whether experimenter sex and participant sex are matched versus mismatched. ${ }^{59}$ However, the absolute importance of matching experimenters with participants according to demographic characteristics such as sex remains debated. A third limitation was that assessment of sleep quality was based on a self-report measure rather than use of objective indicators (ie, actigraphy or polysomnography) to assess aspects of sleep such as sleep efficiency and sleep-onset latency. Additional research addressing sex differences in the effects of sleep on experimental pain responses using both objective and subjective sleep assessment methods represents an interesting topic for future investigation. Finally, it has previously been suggested that variability in the implementation of QST methods, particularly the 
assessment of ischemic pain via the submaximal effort tourniquet test, limits the validity of data generated from such methods. ${ }^{60}$ This study attempted to minimize such variability by using a standardized QST protocol that was consistent with guidelines put forth for the assessment of ischemic pain sensitivity ${ }^{60}$ and CPM. ${ }^{32}$

\section{Conclusion}

Despite its limitations, the present study is helpful for characterizing important differences between men and women in unidimensional measures of pain sensitivity as well as endogenous pain inhibition. Although it has previously been reported that women demonstrate less tolerance for various experimental pain modalities and less-efficient CPM compared with men, we are not aware of any other investigation that has examined sex differences in both unidimensional measures of pain sensitivity as well as dynamic protocols of pain inhibition while accounting for depressive symptoms and sleep quality. It will be important to more directly determine the clinical relevance of these findings in the future. For instance, if additional research continues to reveal that endogenous pain inhibition is predictive of clinical pain outcomes similar to what has already been found, ${ }^{57,58}$ then targeting treatments toward improving pain inhibitory capacity may help reduce the overwhelming burden of chronic pain currently experienced by women.

\section{Acknowledgments}

The study and drafting of this manuscript was supported in part by a Future Leaders in Pain Research award provided to BRG by the American Pain Society, as well as National Institutes of Health (NIH)/ National Center for Advancing Translational Sciences and National Center for Research Resources Clinical and Translational Science Award UL1TR000165 to the University of Alabama at Birmingham. TJN is supported by NIH/National Institute of Diabetes and Digestive and Kidney Diseases (NIDDK) award R01 DK51413, and MTR by NIH/NIDDK awards R00 DK080981, R01 DK100904, and Department of Defense award PR100915. The contents are solely the views of the authors and do not necessarily represent the official views of the NIH.

\section{Disclosure}

The authors report no conflicts of interest in this work.

\section{References}

1. Bouhassira D, Lantéri-Minet M, Attal N, Laurent B, Touboul C. Prevalence of chronic pain with neuropathic characteristics in the general population. Pain. 2008;136(3):380-387.
2. Wijnhoven HA, de Vet HC, Picavet HS. Prevalence of musculoskeletal disorders is systematically higher in women than in men. Clin J Pain. 2006;22(8):717-724.

3. Mogil JS. Sex differences in pain and pain inhibition: multiple explanations of a controversial phenomenon. Nat Rev Neurosci. 2012;13(12):859-866.

4. Bartley EJ, Fillingim RB. Sex differences in pain: a brief review of clinical and experimental findings. Br J Anaesth. 2013;111(1):52-58.

5. Greenspan JD, Craft RM, LeResche L, et al; Consensus Working Group of the Sex, Sex, and Pain SIG of the IASP. Studying sex and sex differences in pain and analgesia: a consensus report. Pain. 2007;132 Suppl 1:S26-S45.

6. Fillingim RB, King CD, Ribeiro-Dasilva MC, Rahim-Williams B, Riley JL 3rd. Sex, sex, and pain: a review of recent clinical and experimental findings. J Pain. 2009;10(5):447-485.

7. Fillingim RB, Edwards RR, Powell T. The relationship of sex and clinical pain to experimental pain responses. Pain. 1999;83(3):419-425.

8. Yarnitsky D. Conditioned pain modulation (the diffuse noxious inhibitory control-like effect): its relevance for acute and chronic pain states. Curr Opin Anaesthesiol. 2010;23(5):611-615.

9. Racine M, Tousignant-Laflamme Y, Kloda LA, Dion D, Dupuis G, Choinière M. A systematic literature review of 10 years of research on sex/sex and experimental pain perception - part 1: are there really differences between women and men? Pain. 2012;153(3):602-618.

10. Popescu A, LeResche L, Truelove EL, Drangsholt MT. Sex differences in pain modulation by diffuse noxious inhibitory controls: a systematic review. Pain. 2010;150(2):309-318.

11. Ge HY, Madeleine P, Arendt-Nielsen L. Sex differences in temporal characteristics of descending inhibitory control: an evaluation using repeated bilateral experimental induction of muscle pain. Pain. 2004;110(1-2):72-78.

12. Goodin BR, McGuire L, Allshouse M, et al. Associations between catastrophizing and endogenous pain-inhibitory processes: sex differences. J Pain. 2009;10(2):180-190.

13. Granot M, Weissman-Fogel I, Crispel Y, et al. Determinants of endogenous analgesia magnitude in a diffuse noxious inhibitory control (DNIC) paradigm: do conditioning stimulus painfulness, sex and personality variables matter? Pain. 2008;136(1-2):142-149.

14. Baad-Hansen L, Poulsen HF, Jensen HM, Svensson P. Lack of sex differences in modulation of experimental intraoral pain by diffuse noxious inhibitory controls (DNIC). Pain. 2005;116(3):359-365.

15. France CR, Suchowiecki S. A comparison of diffuse noxious inhibitory controls in men and women. Pain. 1999;81(1-2):77-84.

16. France CR, Suchowiecki S. Assessing supraspinal modulation of pain perception in individuals at risk for hypertension. Psychophysiology. 2001;38(1):107-113.

17. Locke D, Gibson W, Moss P, Munyard K, Mamotte C, Wright A. Analysis of meaningful conditioned pain modulation effect in a pain-free adult population. J Pain. 2014;15(11):1190-1198.

18. Bair MJ, Robinson RL, Katon W, Kroenke K. Depression and pain comorbidity: a literature review. Arch Intern Med. 2003;163(20):2433-2445.

19. Finan PH, Goodin BR, Smith MT. The association of sleep and pain: an update and a path forward. J Pain. 2013;14(12):1539-1552.

20. Bär KJ, Brehm S, Boettger MK, Boettger S, Wagner G, Sauer H. Pain perception in major depression depends on pain modality. Pain. 2005; 117(1-2):97-103.

21. de Souza JB, Potvin S, Goffaux P, Charest J, Marchand S. The deficit of pain inhibition in fibromyalgia is more pronounced in patients with comorbid depressive symptoms. Clin J Pain. 2009;25(2):123-127.

22. Edwards RR, Grace E, Peterson S, Klick B, Haythornthwaite JA, Smith MT. Sleep continuity and architecture: associations with paininhibitory processes in patients with temporomandibular joint disorder. Eur J Pain. 2009;13(10):1043-1047.

23. Lautenbacher S, Roscher S, Strian D, Fassbender K, Krumrey K, Krieg JC. Pain perception in depression: relationships to symptomatology and naloxone-sensitive mechanisms. Psychosom Med. 1994;56(4): 345-352. 
24. Lautenbacher S, Spernal J, Schreiber W, Krieg JC. Relationship between clinical pain complaints and pain sensitivity in patients with depression and panic disorder. Psychosom Med. 1999;61(6):822-827.

25. Lee YC, Lu B, Edwards RR, et al. The role of sleep problems in central pain processing in rheumatoid arthritis. Arthritis Rheum. 2013;65(1): 59-68.

26. Smith MT, Edwards RR, McCann UD, Haythornthwaite JA. The effects of sleep deprivation on pain inhibition and spontaneous pain in women. Sleep. 2007;30(4):494-505.

27. Goodin BR, Anderson AJ, Freeman EL, Bulls HW, Robbins MT, Ness TJ. Intranasal oxytocin administration is associated with enhanced endogenous pain inhibition and reduced negative mood states. Clin J Pain. Epub November 3, 2014.

28. Posner J. A modified submaximal effort tourniquet test for evaluation of analgesics in healthy volunteers. Pain. 1984;19(2):143-151.

29. Jensen MP, Karoly P. Self-report scales and procedures for assessing pain in adults. In: Turk DC, Melzack R, editors. Handbook of Pain Assessment. New York, NY: Guilford Press, 1992:135-151.

30. Edens JL, Gil KM. Experimental induction of pain: utility in the study of clinical pain. Behav Ther. 1995;26(2):197-216.

31. Pud D, Granovsky Y, Yarnitsky D. The methodology of experimentally induced diffuse noxious inhibitory control (DNIC)-like effect in humans. Pain. 2009;144(1-2):16-19.

32. Yarnitsky D, Arendt-Nielsen L, Bouhassira D, et al. Recommendations on terminology and practice of psychophysical DNIC testing. Eur $J$ Pain. 2010;14(4):339.

33. Radloff LS. The CES-D scale: a self-report depression scale for research in the general population. Appl Psychol Meas. 1977;1(3):385-401.

34. Devins GM, Orme CM, Costello CG, et al. Measuring depressive symptoms in illness populations: psychometric properties of the Center for Epidemiologic Studies Depression (CES-D) scale. Psychol Health. 1988;2(2):139-156.

35. Buysse DJ, Reynolds CF 3rd, Monk TH, Berman SR, Kupfer DJ. The Pittsburgh Sleep Quality Index: a new instrument for psychiatric practice and research. Psychiatry Res. 1989;28(2):193-213.

36. Backhaus J, Junghanns K, Broocks A, Riemann D, Hohagen F. Testretest reliability and validity of the Pittsburgh Sleep Quality Index in primary insomnia. J Psychosom Res. 2002;53(3):737-740.

37. Riley JL, Robinson ME, Wise EA, Myers CD, Fillingim RB. Sex differences in the perception of noxious experimental stimuli: a metaanalysis. Pain. 1998;74(2-3):181-187.

38. Nir RR, Granovsky Y, Yarnitsky D, Sprecher E, Granot M. A psychophysical study of endogenous analgesia: the role of the conditioning pain in the induction and magnitude of conditioned pain modulation. Eur J Pain. 2011;15(5):491-497.

39. Gioiosa L, Chen X, Watkins R, et al. Sex chromosome complement affects nociception in tests of acute and chronic exposure to morphine in mice. Horm Behav. 2008;53(1):124-130.

40. Borzan J, Fuchs PN. Organizational and activational effects of testosterone on carrageenan-induced inflammatory pain and morphine analgesia. Neuroscience. 2006;143(3):885-893.

41. Dina OA, Aley KO, Isenberg W, Messing RO, Levine JD. Sex hormones regulate the contribution of PKCepsilon and PKA signalling in inflammatory pain in the rat. Eur $J$ Neurosci. 2001;13(12): 2227-2233.

42. Hucho TB, Dina OA, Kuhn J, Levine JD. Estrogen controls PKCepsilondependent mechanical hyperalgesia through direct action on nociceptive neurons. Eur J Neurosci. 2006;24(2):527-534.
43. Craft RM. Modulation of pain by estrogens. Pain. 2007;132 Suppl 1: S3-S12.

44. Smith YR, Stohler CS, Nichols TE, Bueller JA, Koeppe RA, Zubieta JK. Pronociceptive and antinociceptive effects of estradiol through endogenous opioid neurotransmission in women. J Neurosci. 2006;26(21): 5777-5785.

45. Sorge RE, LaCroix-Fralish ML, Tuttle AH, et al. Spinal cord Toll-like receptor 4 mediates inflammatory and neuropathic hypersensitivity in male but not female mice. J Neurosci. 2011;31(43):15450-15454.

46. Aloisi AM, Affaitati G, Ceccarelli I, et al. Estradiol and testosterone differently affect visceral pain-related behavioural responses in male and female rats. Eur J Pain. 2010;14(6):602-607.

47. Linnman C, Beucke JC, Jensen KB, Gollub RL, Kong J. Sex similarities and differences in pain-related periaqueductal gray connectivity. Pain. 2012;153(2):444-454.

48. Loyd DR, Murphy AZ. The role of the periaqueductal gray in the modulation of pain in males and females: are the anatomy and physiology really that different? Neural Plast. 2009;2009:462879.

49. Dickens C, McGowan L, Dale S. Impact of depression on experimental pain perception: a systematic review of the literature with meta-analysis. Psychosom Med. 2003;65(3):369-375.

50. Haack M, Scott-Sutherland J, Santangelo G, Simpson NS, Sethna N, Mullington JM. Pain sensitivity and modulation in primary insomnia. Eur J Pain. 2012;16(4):522-533.

51. Paller CJ, Campbell CM, Edwards RR, Dobs AS. Sex-based differences in pain perception and treatment. Pain Med. 2009;10(2):289-299.

52. Racine M, Tousignant-Laflamme Y, Kloda LA, Dion D, Dupuis G, Choinière M. A systematic literature review of 10 years of research on sex/sex and pain perception - part 2: do biopsychosocial factors alter pain sensitivity differently in women and men? Pain. 2012;153(3): 619-635.

53. Manocchia M, Keller S, Ware JE. Sleep problems, health-related quality of life, work functioning and health care utilization among the chronically ill. Qual Life Res. 2001;10(4):331-345.

54. Moussavi S, Chatterji S, Verdes E, Tandon A, Patel V, Ustun B. Depression, chronic diseases, and decrements in health: results from the World Health Surveys. Lancet. 2007;370(9590):851-858.

55. Edwards RR, Sarlani E, Wesselmann U, Fillingim RB. Quantitative assessment of experimental pain perception: multiple domains of clinical relevance. Pain. 2005;114(3):315-319.

56. Edwards RR, Doleys DM, Lowery D, Fillingim RB. Pain tolerance as a predictor of outcome following multidisciplinary treatment for chronic pain: differential effects as a function of sex. Pain. 2003;106(3): 419-426.

57. Edwards RR, Ness TJ, Weigent DA, Fillingim RB. Individual differences in diffuse noxious inhibitory controls (DNIC): association with clinical variables. Pain. 2003;106(3):427-437.

58. Yarnitsky D, Crispel Y, Eisenberg E, et al. Prediction of chronic postoperative pain: pre-operative DNIC testing identifies patients at risk Pain. 2008;138(1):22-28.

59. Kállai I, Barke A, Voss U. The effects of experimenter characteristics on pain reports in women and men. Pain. 2004;112(1-2):142-147.

60. Moore PA, Duncan GH, Scott DS, Gregg JM, Ghia JN. The submaximal effort tourniquet test: its use in evaluating experimental and chronic pain. Pain. 1979;6(3):375-382. 
Journal of Pain Research

\section{Publish your work in this journal}

The Journal of Pain Research is an international, peer-reviewed, open access, online journal that welcomes laboratory and clinical findings in the fields of pain research and the prevention and management of pain. Original research, reviews, symposium reports, hypothesis formation and commentaries are all considered for publication.

The manuscript management system is completely online and includes a very quick and fair peer-review system, which is all easy to use. Visit http://www.dovepress.com/testimonials.php to read real quotes from published authors.

\footnotetext{
Submit your manuscript here: http://www.dovepress.com/journal-of-pain-research-journal
} 